\title{
Psychometric Properties of the Swedish Version of the Fear of Complications Questionnaire
}

\author{
Marie Olsen", Therese Anderbro², Susanne Amsberg',3, Janeth Leksell1,4, Erik Moberg5, \\ Jan Lisspers ${ }^{6}$, Soffia Gudbjörnsdottir ${ }^{7}$, Unn-Britt Johansson ${ }^{3,8}$ \\ ${ }^{1}$ Dalarna University, Department of Health and Social Studies, Falun, Sweden \\ ${ }^{2}$ Karolinska Institutet, Department of Clinical Sciences, Danderyd Hospital, Division of Medicine, \\ Stockholm, Sweden \\ ${ }^{3}$ Sophiahemmet University, Stockholm, Sweden \\ ${ }^{4}$ Uppsala University, Department of Medical Sciences, Uppsala, Sweden \\ ${ }^{5}$ Karolinska Institutet, Department of Medicine, Karolinska University Hospital, Huddinge, Sweden \\ ${ }^{6}$ Mid Sweden University, Department of Psychology, Campus Östersund, Östersund, Sweden \\ ${ }^{7}$ University of Gothenburg, Department of Medicine, Sahlgrenska University Hospital, Gothenburg, Sweden \\ ${ }^{8}$ Karolinska Institutet, Department of Clinical Science and Education, Södersjukhuset, Stockholm, Sweden \\ Email: therese.anderbro@ki.se
}

Received 17 February 2014; revised 17 March 2014; accepted 24 March 2014

Copyright (C) 2014 by authors and Scientific Research Publishing Inc.

This work is licensed under the Creative Commons Attribution International License (CC BY).

http://creativecommons.org/licenses/by/4.0/

(c) (i) Open Access

\section{Abstract}

Objectives: To translate and evaluate the psychometric properties of the Swedish version of the Fear of Complications Questionnaire. Design: Cross-sectional study design and scale development. Settings: Totally, 469 adults (response rate 63.5\%) with Type 1 diabetes completed the questionnaires. Participants were recruited from two university hospitals in Sweden. Participants: Eligible patients were those who met the following inclusion criteria: diagnosed with Type 1 diabetes, diabetes duration of at least 1 year and aged at least 18 years. Methods: The Fear of Complications Questionnaire was translated using the forward-backward translation method. Factor analyses of the questionnaire were performed in two steps using both exploratory and confirmatory factor analysis. Convergent validity was examined using the Hospital Anxiety and Depression Scale and the Fear of Hypoglycaemia Fear Survey. Internal consistency was estimated using Cronbach's alpha. Results: Exploratory factor analysis supported a two-factor solution. One factor contained three items having to do with fear of kidney-related complications and one factor included the rest of items concerning fear of other diabetes-related complications, as well as fear of complications in general. Internal consistency was high Cronbach's alpha 0.96. The findings also gave support for convergent validity, with significant positive correlations between measures $(r=0.51$ to 0.54$)$. Conclusion: The clinical relevance of the identified two-factor model with a structure of one do- 
minant subdomain may be considered. We suggest, however a one-factor model covering all the items as a relevant basis to assess fear of complications among people with Type 1 diabetes.

\title{
Keywords
}

\author{
Diabetes Mellitus, Diabetes Complications, Factor Analysis, Fear, Psychometrics
}

\section{Introduction}

Patients' own experience of living with a chronic condition and its treatment is a major indicator of quality of care [1]. Recently, a multinational study showed that it is important to capture an individual's barriers for achieving optimal diabetes self-care in order to attain a good glucose level and enhance quality of life (QoL) [2]. Maintaining a good glucose control level is a major task among persons with diabetes [3] and is a balancing act [4] in relation to the individual's QoL [5]. Failure to maintain a good glucose control level puts the person at risk of developing future diabetes-related microvascular complications (e.g., neuropathy, retinopathy and nephropathy) and macrovascular complications (e.g., cardiovascular disease and stroke) [6] [7]. On the other hand, an overly strict blood glucose control level may increase the risk for hypoglycaemic events and immediate unpleasant symptoms [8] [9]. The constant threat of hyperglycaemia and hypoglycaemia is complex, where the person with diabetes can be trapped between fear of diabetes complications and fear of hypoglycaemia [10]. It is well known that fear of hypoglycaemia may have significant clinical implications for diabetes self-care. Previous research on fear of hypoglycaemia has reported about its measurement, predictors, correlates and impact as well as treatment [9]. It is also well known that diabetes-related complications have the greatest perceived burden on QoL among persons with diabetes [5] [11] [12]. Diabetes-related complications have been shown to be a predictor of anxiety and are associated with depression [13]-[16]. A review of the literature describing various problem areas in diabetes shows that a major concern among many persons with diabetes is future complications of diabetes [2] [17]-[21]. Furthermore, studies on emotional aspects of late complications of diabetes are scarce. Both national and international guidelines stress the importance of addressing psychological aspects in diabetes care [22]-[24]. This facilitates the provision of good support and individualized care and thus enables patients to manage their diabetes optimally. Fear of complication has been shown to be a major problem area among persons with diabetes. Accordingly, to provide optimal support and care it is essential to use a well-validated scale that measures this type of fear. This study aimed to evaluate the psychometric properties of the Swedish version of the Fear of Complications Questionnaire (FCQ).

\section{Methods}

\subsection{Design and Sample}

This study was a cross-sectional survey with a quantitative approach. The study sample consisted of patients with Type 1 diabetes with diabetes duration of 1 year or more. The patients from two university hospitals in Stockholm, Sweden were aged 18 years or older. Written study information was sent to 764 patients with Type 1 diabetes together with the questionnaire and information about voluntary participation. A pre-paid return envelope was also included. Two weeks after the initial mailing, a remainder letter was mailed to those patients who had not returned the questionnaires. Data on age, sex, $\mathrm{HbA}_{1 \mathrm{c}}$ and diabetes duration were collected from the patients' medical records. This study was part of a cross-sectional survey conducted to investigate fear of hypoglycaemia [17].

\subsection{Measures}

\subsubsection{Fear of Complications Questionnaire}

The FCQ is a 15 item self-assessment questionnaire designed to measure fear of complications in Type 1 diabetes. The 15 items are rated on a four-point scale $(0-3)$, where 0 denotes low fear of complications and 3 refers to high fear. Cronbach's alpha was 0.94 for the total scale [5]. 


\subsubsection{Hospital Anxiety and Depression Scale}

The original Hospital Anxiety and Depression Scale (HADS), which measures anxiety and depression was developed by Zigmond and Snaith [25]. The HADS consists of 14 items of which 7 relate to anxiety and 7 to depression. Responses are rated on a four-point Likert scale score $(0-3)$, where 0 represents the lowest level of anxiety and depression and 3 the highest level. The Swedish version of HADS has been used in this study. Cronbach's alpha was 0.90 for the total HADS scale [26]

\subsubsection{Fear of Hypoglycaemia Survey}

The original Fear of Hypoglycaemia Survey (HFS) was designed to measure fear of hypoglycaemia in adults with Type 1 diabetes [27] [28]. The HFS comprises 23 items rated on a five-point Likert scale resulting in a total sum score ranging from 0 to 92 with higher scores indicating a greater fear of hypoglycaemia. The HFS has been translated into Swedish and psychometrically evaluated for Swedish conditions in people with Type 1 diabetes. Cronbach’s alpha for the entire scale was 0.85 [29].

\subsection{Translation Process}

The original FCQ was translated into Swedish using the forward-backward translation method. We closely followed the steps for test adaption according to Streiner et al. [30]. The procedure involves four steps: forward translation, expert panel discussions, backward translation and a panel discussion with a review and consensus on the final version.

A certified translator performed the initial forward translation by translating the original FCQ from English into Swedish. The expert panel consisting of four researchers: two psychologists, one medical doctor and one nurse, the doctor and nurse were specialised in diabetes. The expert panel discussed the forward translation by identifying inadequate expressions or concepts. The questionnaire was back-translated and the expert panel examined the translation for quality control to ensure that the original meaning had not been lost in translation. Finally a few changes were made after the expert panel discussions.

\subsection{Statistical Analyses}

Statistical analyses were performed using R 2.14. (R Statistical Computing, Vienna, Austria) and SPSS version 20 for Windows (IBM, SPSS, NY, USA). The exploratory factor analyses were conducted using the factorial function in the base package; the confirmatory factor analyses were performed using the sem function in the sem package (Structural Equation Models, R package version 2.1-1).The factor analyses on the FCQ were carried out in two steps. In the first step an exploratory factor analysis (EFA) was performed on the results from a randomly selected sample of half of the patients $(n=234)$. In the EFA the models were fitted by a maximum likelihood method and the resulting factors were rotated using varimax rotation procedure. Based on the results from the EFA, a confirmatory factor analysis (CFA) was fitted for the results obtained from the other half of the patients $(n=233)$. The chi-square test was applied to assess the overall goodness-of-fit of the CFA. Furthermore, the comparative fit index (CFI), the standardized root mean squared residual (SRMR) and the root mean square error of approximation (RMSEA) were estimated. The criterion values for the CFI values range from 0 - 1, with a minimum of goodness-of-fit value of 0.90 (acceptable) and $>0.95$ (excellent). A Model with a good fit a RMSEA index between 0.00 and 0.05 [31]. For SRMR, a criterion value close to 0.08 or below is recommended [32]. The input correlation matrix for the confirmatory factor analyses was based on Pearson's correlation coefficients. Pairwise deletion was used to deal with missing values. Using Pearson correlations, we assessed convergent validity to determine the relationship between the FCQ total scale with the Swe-HFS and HADS. The hypothesis was that people who rate a high degree of anxiety and depression are also likely to rate a high degree of fear of complications. Moreover, people who rate a high degree of fear of hypoglycaemia are expected to rate a high degree of fear of complications. Internal consistency was measured by Cronbach's alpha coefficient; a value of 0.70 is generally considered acceptable [33].

\subsection{Ethical Considerations}

Ethical approval was obtained for the study from the Regional Ethics Committee, Karolinska. Institutet, Stockholm, Sweden. The participants were promised anonymity and confidentiality. All participants 
gave their written informed consent.

\section{Results}

The sample comprised 469 adults with Type 1 diabetes (49\% males) who had a mean age of 47 years (SD 14.0). Mean duration of diabetes was 31.0 years (SD 14.2) and mean HbA1c was 6.9\% (SD 1.0). The response rate was $63.5 \%$.

\subsection{Exploratory Factor Analysis}

Exploratory factor analysis (EFA) and the scree plot supported a two-factor solution with an eigenvalue greater than 1 (Figure 1). Factor loadings using principal component extraction with varimax rotation are presented in Table 1 . The analysis showed that in total these two factors accounted for $0.62 \%$ of the variance in the 15 -item scale. The first factor included items of general fear of complications and fear of complications for heart, blood circulation, feet, eyes and head (stroke). The other factor was restricted to kidney complications. Factor loadings for the one-factor solution ( 0.682 - 0.819$)$ as suggested by Taylor et al. [5] accounted for $0.56 \%$ of the variance in the 15 -item scale.

\subsection{Confirmatory Factor Analysis}

Confirmatory factor analysis for the FCQ showed no clear support or a two-factor solution (SRMR 0.25), but did indicate a one-factor solution (SRMR 0.08) (Table 2).

\subsection{Convergent Validity}

To examine convergent validity the FCQ was compared with other validated scales. Pearson's correlations showed that the FCQ was significantly positively correlated with the Swe-FHS (fear of hypoglycaemia) and the HADS and Anxiety and Depression sub-scales $(p<0.001)$ (Table 3).

\subsection{Internal Consistency}

Cronbach's alpha coefficients for the FCQ 15-item scale $(n=467)$ was 0.956 (95\% CI0.943:0.967).

\section{Discussion}

The results of the EFA suggest a two-factor model as optimal for the FCQ, with the first factor measuring gen-

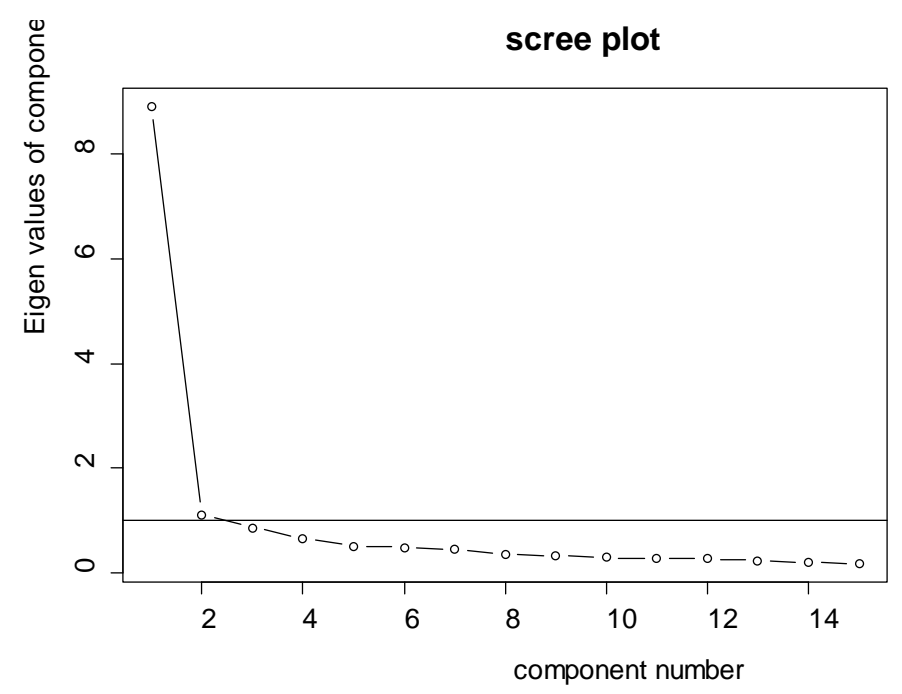

Figure 1. Factor components for the fear of complications questionnaire $(n=234)$. 
Table 1. Factor analysis with two-factors and varimax rotation.

\begin{tabular}{lcc}
\hline & Factor 1 & Factor 2 \\
\hline FCQ 1 & 0.686 & \\
FCQ 3 & 0.764 & \\
FCQ 4 & 0.634 & 0.757 \\
FCQ 5 & & 0.817 \\
FCQ 6 & 0.715 & 0.783 \\
FCQ 7 & & \\
FCQ 8 & & \\
FCQ 9 & 0.665 & \\
FCQ 10 & 0.603 & \\
FCQ 11 & 0.659 & \\
FCQ 12 & 0.758 & \\
FCQ 13 & 0.648 & \\
FCQ 14 & 0.681 & \\
FCQ 15 & 0.696 & \\
\hline
\end{tabular}

Cumulative variance: 0.622 .

Table 2. Fit indices for the confirmatory factor analysis.

\begin{tabular}{cccccc}
\hline Model & $\mathbf{X}^{2}$ & df & CFI & SRMR & RMSEA \\
\hline Single-factor model & 617.1 & 90 & 0.77 & 0.08 & 0.16 \\
Two-factor model & 502.6 & 90 & 0.82 & 0.14 & 0.33 \\
Three-factor model & 536.1 & 90 & 0.15 \\
\hline
\end{tabular}

CFI = comparative fit index, SRMR = standardised root mean squared residual, RMSEA = root mean square error of approximation.

Table 3. Pearson's correlation coefficients.

\begin{tabular}{|c|c|c|c|c|c|}
\hline & \multirow{2}{*}{ FCQ } & \multirow{2}{*}{ HADS } & HADS & HADS & \multirow{2}{*}{ HFS } \\
\hline & & & Anxiety & Depression & \\
\hline FCQ & 1 & 0.54 & 0.55 & 0.44 & 0.51 \\
\hline HADS & $0.54^{*}$ & 1 & 0.91 & 0.91 & 0.48 \\
\hline $\begin{array}{l}\text { HADS } \\
\text { Anxiety }\end{array}$ & $0.55^{*}$ & 0.91 & 1 & 0.66 & 0.48 \\
\hline $\begin{array}{c}\text { HADS } \\
\text { Depression }\end{array}$ & $0.44^{*}$ & 0.91 & 0.66 & 1 & 0.38 \\
\hline HFS & $0.51^{*}$ & 0.48 & 0.48 & 0.38 & 1 \\
\hline
\end{tabular}

" $\mathrm{p}$-value $<0.0001$, FCQ = Fear of Complications Questionnaire, HADs = Hospital Anxiety and Depression Scale, HFS= Fear of Hypoglycaemia Survey.

eral fear of complications and the second measuring fear of kidney complications. However, the follow-up CFA did not find a clear support for a two-factor model. Instead, a one-factor model had a better fit, a finding consistent with that of the original study evaluating FCQ [5].

Reasons for the discrepancy between the EFA and CFA may stem from how the EFA was interpreted. EFA is a statistical method in which several alternatives are available to determine the number of factors. Our use of an eigenvalue $\geq 1$ as a criterion for factor extraction may be questioned as this is considered by many to be the least accurate method of selecting factors [34] because it often leads to too many factors being selected. To examine 
the screen plot, for the natural bend is perhaps a better criterion. However, this result can also be questioned in that the second factor is very close to the line. A better way to determine the number of factors would probably have been to use parallel analysis. With this in mind, we propose like the original authors [5], a one-factor model covering all items as a relevant basis for assessing fear of complications among persons with Type 1 diabetes. It offers a useful tool to approach and discuss these issues among patients.

The reliability findings of this study are consistent with the original evaluation of the FCQ [5]. Statistical analyses gave support for the internal consistency of the instrument.

A significant correlation was found between fear of complications and anxiety and depression as measured with the HADS [27]. Fear of complications was also significant correlated to fear of hypoglycaemia [29] in the domains of worry, avoidance and aloneness.

The moderately significant correlations between fear of complications and fear of hypoglycaemia and more symptoms of depression and anxiety are concept-related as expected, which provides evidence of convergent validity. Finding reveals a need for research that focuses on understanding the mechanisms of fear itself in people with diabetes. We also speculate upon the need for items that reflect the personal meaning of fear of complications, i.e. consequences in behaviour that appear because of the fear, such as constantly maintaining a very low blood sugar level excessive use of self-monitoring of blood glucose or patient refusal to self-monitor blood glucose.

The FCQ can determine general fear among persons with diabetes; fear can manifest itself in various forms, which in turn can affect how people with diabetes perform their self-care. It is therefore important in clinical practice to screen for fear of complications to determine the patients' general fear related to their disease. Taking this into consideration, it seems meaningful to speculate upon the need for further research in this area. For instance, a qualitative approach would contribute to a deeper understanding of the phenomenon as well as to contribute to clinicians' improved understanding of the problem.

Further work is necessary to ensure the questionnaire's applicability to people with Type 2 diabetes and its treatment regimen. Our overall, results suggest that the Swedish version of the FCQ has satisfactory psychometric properties for mapping general fear of complications in people with Type 1 diabetes.

\section{Conclusion}

The clinical relevance of the identified two-factor model with a structure of one dominant subdomain may be considered. We suggest, however a one-factor model covering all the items as a relevant basis to assess fear of complications among people with Type 1 diabetes.

\section{Acknowledgements}

We would like to acknowledge Marcus Thuresson for his support and assistance with the statistical analysis. This study was funded by Sophiahemmet University, Sophiahemmet Foundation, in Stockholm and Diabetes fonden. The authors declare that they have no conflicts of interest.

\section{References}

[1] Haywood, K., Marshall, S. and Fitzpatrick, R. (2006) Patient Participation in the Consultation Process: A Structured Review of Intervention Strategies. Patient Education and Counseling, 63, 12-23. http://dx.doi.org/10.1016/j.pec.2005.10.005

[2] Snoek, F.J. (2000) Barriers to Good Glycaemic Control: The Patient’s Perspective. International Journal of Obesity, 24, S12-S20. http://dx.doi.org/10.1038/sj.ijo.0801421

[3] Morrison, F., Shubina, M. and Turchin, A. (2012) Lifestyle Counseling in Routine Care and Long-Term Glucose, Blood Pressure, and Cholesterol Control in Patients with Diabetes. Diabetes Care, 35, 334-341. http://dx.doi.org/10.2337/dc11-1635

[4] Paterson, B.L., Thorne, S. and Dewis, M. (2007) Adapting to and Managing Diabetes. Journal of Nursing Scholarship, 30, 57-62. http://dx.doi.org/10.1111/j.1547-5069.1998.tb01237.x

[5] Taylor, E.P., Crawford, J.R. and Gold, A.E. (2005) Design and Development of a Scale Measuring Fear of Complications in Type 1 Diabetes. Diabetes-Metabolism Research and Reviews, 21, 264-270. http://dx.doi.org/10.1002/dmrr.524

[6] DDCT (1997) Hypoglycemia in the Diabetes Control and Complications Trial. The Diabetes Control and Complica- 
tions Trial Research Group. Diabetes, 46, 271-286. http://dx.doi.org/10.2337/diab.46.2.271

[7] Swedish Council on Technology Assessment in Health Care (SBU) (2009) Intensive Glucose-Lowering Therapy in Diabetes (Summary and Conclusions) Systematic Review. Report No: 196.

http://www.sbu.se/upload/Publikationer/Content1/1/Intensive_glucose_summary.pdf

[8] Irvine, A.A., Cox, D. and Gonder-Frederick, L. (1992) Fear of Hypoglycemia-Relationship to Physical and Psychological Symptoms in Patients with Insulin-Dependent Diabetes-Mellitus. Health Psychology, 11, 135-138. http://dx.doi.org/10.1037//0278-6133.11.2.135

[9] Wild, D., von Maltzahn, R., Brohan, E., Christensen, T., Clauson, P. and Gonder-Frederick, L. (2007) A Critical Review of the Literature on Fear of Hypoglycemia in Diabetes: Implications for Diabetes Management and Patient Education. Patient Education and Counseling, 68, 10-15. http://dx.doi.org/10.1016/j.pec.2007.05.003

[10] Shiu, A.T. and Wong, R.Y. (2002) Fears and Worries Associated with Hypoglycaemia and Diabetes Complications: Perceptions and Experience of Hong Kong Chinese Clients. Journal of Advanced Nursing, 39, 155-163. http://dx.doi.org/10.1046/j.1365-2648.2002.02255.X

[11] Akinci, F., Yildirim, A., Gozu, H., Sargin, H., Orbay, E. and Sargin, M. (2008) Assessment of Health-Related Quality of Life (HRQoL) of Patients with Type 2 Diabetes in Turkey. Diabetes Research and Clinical Practice, 79, 117-123. http://dx.doi.org/10.1016/j.diabres.2007.07.003

[12] Solli, O., Stavem, K. and Kristiansen, I.S. (2010) Health-Related Quality of Life in Diabetes: The Associations of Complications with EQ-5D Scores. Health and Quality of Life Outcomes, 8, 18. http://dx.doi.org/10.1186/1477-7525-8-18

[13] Peyrot, M., Rubin, R.R., Lauritzen, T., Snoek, F.J., Matthews, D.R. and Skovlund S.E. (2005) Psychosocial Problems and Barriers to Improved Diabetes Management: Results of the Cross-National Diabetes Attitudes, Wishes and Needs (DAWN) Study. Diabetic Medicine. 22, 1379-1385. http://dx.doi.org/10.1111/j.1464-5491.2005.01644.x

[14] Saatci, E., Tahmiscioglu, G., Bozdemir, N., Akpinar, E., Ozcan, S. and Kurdak, H. (2010) The Well-Being and Treatment Satisfaction of Diabetic Patients in Primary Care. Health and Quality of Life Outcomes, 8, 67. http://dx.doi.org/10.1186/1477-7525-8-67

[15] Sulaiman, N., Hamdan, A., Tamim, H., Mahmood, D.A. and Young, D. (2010) The Prevalence and Correlates of Depression and Anxiety in a Sample of Diabetic Patients in Sharjah, United Arab Emirates. BMC Family Practice, 11, 80. http://dx.doi.org/10.1186/1471-2296-11-80

[16] Wu, S.F.V., Huang, Y.C., Liang, S.Y., Wang, T.J., Lee, M.C. and Tung, H.H. (2011) Relationships among Depression, Anxiety, Self-Care Behaviour and diabetes Education Difficulties in Patients with Type-2 Diabetes: A Cross-Sectional Questionnaire Survey. International Journal of Nursing Studies, 48, 1376-1383. http://dx.doi.org/10.1016/j.ijnurstu.2011.04.008

[17] Anderbro, T. (2012) Behavior Change Intervention and Fear of Hypoglycemia in Type 1 Diabetes. Doctoral thesis, Karolinska Institutet., Stockholm. http://publications.ki.se/xmlui/bitstream/handle/10616/41244/Thesis_Therese_Anderbro.pdf?sequence=1

[18] Heuer, L. and Lausch, C. (2006) Living with diabetes: Perceptions of Hispanic migrant farmworkers. Journal of Community Health Nursing, 23, 49-64. http://dx.doi.org/10.1207/s15327655jchn2301_5

[19] Huang, E.S., Brown, S.E., Ewigman, B.G., Foley, E.C. and Meltzer, D.O. (2007) Patient Perceptions of Quality of Life with Diabetes-Related Complications and Treatments. Diabetes Care, 30, 2478-2483. http://dx.doi.org/10.2337/dc07-0499

[20] Huis In‘T Veld, E.M., Makine, C., Nouwen, A., Karsidag, C., Kadioglu, P., Karsidag, K. and Pouwer, F. (2011) Validation of the Turkish Version of the Problem Areas in Diabetes Scale. Cardiovascular Psychiatry Neurology, 2011, Article ID: 315068. http://dx.doi.org/10.1155/2011/315068

[21] Papadopoulos, A.A., Kontodimopoulos, N., Frydas, A., Ikonomakis, E. and Niakas, D. (2007) Predictors of Health-Related Quality of Life in Type II Diabetic Patients in Greece. BMC Public Health, 7, 186. http://dx.doi.org/10.1186/1471-2458-7-186

[22] International Diabetes Federation (2012) Global Guideline for Type 2 Diabetes: Clinical Guidelines Task Force. http://www.idf.org/sites/default/files/IDF-Guideline-for-Type-2-Diabetes.pdf

[23] The National Board of Health and Welfare (Socialstyrelsen) (2010) National Guidelines for Diabetes Care—-Support for Governance and Management. The National Board of Health and Welfare, p. 128. http://www.socialstyrelsen.se/publikationer2010/2010-2-2

[24] American Diabetes Association (2012) Standards of Medical Care in Diabetes_2012. Diabetes Care, 35, 11-63. http://dx.doi.org/10.2337/dc12-s011

[25] Zigmond, A.S. and Snaith, R.P. (1983) The Hospital Anxiety and Depression Scale. Acta Psychiatrica Scandinavia, 67, 
361-370. http://dx.doi.org/10.1111/j.1600-0447.1983.tb09716.x

[26] Lisspers, J., Nygren, A. and Soderman, E. (1997) Hospital Anxiety and Depression Scale (HAD): Some Psychometric Data for a Swedish Sample. Acta Psychiatrica Scandinavia, 96, 281-286. http://dx.doi.org/10.1111/j.1600-0447.1997.tb10164.x

[27] Cox, D.J., Irvine, A., Gonder-Frederick, L., Nowacek, G. and Butterfield, J. (1987) Fear of Hypoglycemia: Quantification, Validation, and Utilization. Diabetes Care, 10, 617-621. http://dx.doi.org/10.2337/diacare.10.5.617

[28] Gonder-Frederick, L.A., Schmidt, K.M., Vajda, K.A., Greear, M.L., Singh, H., Shepard, J.A. and Cox, D.J. (2011) Psychometric Properties of the Hypoglycemia Fear Survey-II for Adults with Type 1 Diabetes. Diabetes Care, 34, 801806. http://dx.doi.org/10.2337/dc10-1343

[29] Anderbro, T., Amsberg, S., Wredling, R., Lins, P.E., Adamson, U., Lisspers, J. and Johansson, U.B. (2008) Psychometric Evaluation of the Swedish Version of the Hypoglycaemia Fear Survey. Patient Education and Counseling, 73, 127-131. http://dx.doi.org/10.1016/j.pec.2008.03.022

[30] Streiner, D. and Norman, G. (2008) Health Measurement Scales—A Practical Guide to Their Development and Use. 4th Edition, Oxford University Press Inc., New York.

[31] Cudeck, R.Y. and Browne, M.W. (1983) Cross-Validation of Covariance Structures. Multivariate Behavioral Research, 18, 47-167.

[32] Hu, L.T. and Bentler, P.M. (1999) Cutoff Criteria for Fit Indexes in Covariance Structure Analysis: Conventional Criteria Versus New Alternatives. Structural Equation Modeling-A Multidisciplinary Journal, 6, 1-55. http://dx.doi.org/10.1080/10705519909540118

[33] Nunnally, J.C. and Bernstein, I.H. (1994) Psychometric Theory. 3rd Edition, McGraw-Hill, New York.

[34] Velicer, W.F. and Jackson, D.N. (1990) Component Analysis versus Common Factor Analysis: Some Further Observations. Multivariate Behavioral Research, 25, 97-114. http://dx.doi.org/10.1207/s15327906mbr2501_12 